\title{
WA Notify: the planning and implementation of a Bluetooth exposure notification tool for COVID-19 pandemic response in Washington State
}

\author{
Tiffany Chen ${ }^{1}$, Janet Baseman ${ }^{1 *}$, William B. Lober ${ }^{2}$, Debra Revere ${ }^{3}$, Rebecca Hills ${ }^{1}$, Nola \\ Klemfuss ${ }^{4}$, Bryant T. Karras ${ }^{5}$ \\ ${ }^{1}$ Department of Epidemiology, School of Public Health, University of Washington, Seattle, WA \\ ${ }^{2}$ Biobehavioral Nursing \& Health Informatics, School of Nursing, University of Washington, Seattle, WA \\ ${ }^{3}$ Department of Health Services, School of Public Health, University of Washington, Seattle, WA \\ ${ }^{4}$ Brotman Baty Institute for Precision Medicine, School of Medicine, University of Washington, Seattle, \\ WA
}

${ }^{5}$ Office of Science, Health and Informatics, Washington State Department of Health, Olympia, WA

\begin{abstract}
Bluetooth exposure notification tools for mobile phones have emerged as one way to support public health contact tracing and mitigate the spread of COVID-19. Many states have launched their own versions of these tools. Washington State's exposure notification tool, WA Notify, became available on November 30, 2020, following a one-month Seattle campus pilot at the University of Washington. By the end of April 2021, 25\% of the state's population had activated WA Notify, one of the highest adoption rates in the country. Washington State's formation of an Exposure Notification Advisory Committee, early pilot testing, and use of the EN Express system framework were all important factors in its adoption. Continuous monitoring and willingness to make early adjustments such as switching to automated texting of verification codes have also been important for improving the tool's value. Evaluation work is ongoing to determine and quantify WA Notify's effectiveness, timeliness, and accessibility.

Keywords: COVID-19, Smartphone, Mobile Applications, Contact Tracing, Communicable Disease Control

Abbreviations: Association of Public Health Laboratories (APHL), Bluetooth Low Energy (BLE), Case Risk Exposure and Risk Surveillance Tool (CREST), Department of Health (DOH), Electronic Lab Reportable (ELR), Environmental Health and Safety (EH\&S), Exposure Notification (EN), EN Express (ENX), Google|Apple exposure notification (GAEN), Local Health Jurisdiction (LHJ), University of Washington (UW), Washington State (WA State)

DOI: 10.5210/ojphi.v13i1.11694

Copyright (C2021 the author(s)

This is an Open Access article. Authors own copyright of their articles appearing in the Online Journal of Public Health Informatics. Readers may copy articles without permission of the copyright owner(s), as long as the author and OJPHI are acknowledged in the copy and the copy is used for educational, not-for-profit purposes.
\end{abstract}




\section{Introduction}

Contact tracing and case investigation have been some of the most essential tools to control the spread of infectious diseases, beginning as early as the late $19^{\text {th }}$ century [1]. During a case interview, patients with confirmed or suspected cases of an illness are given guidance and support on what to do next and interviewed about who they may have been in contact with. These contacts are then "traced" and informed of their exposure for them to take protective measures such as quarantine [2]. In modern times, contact tracing is commonly used for diseases like tuberculosis, measles, and sexually transmitted diseases such as HIV. Contact tracing has been important for controlling outbreaks of diseases like Ebola and smallpox, and novel viruses such as H1N1 in the past [3-5].

Rapid contact tracing is one of public health's most effective tools in limiting transmission of SARS-CoV-2, however, contact tracing personnel require specialized training. Existing contact tracing workforce and public health infrastructure have struggled to support the large numbers of cases, leading many states and countries to hire and train thousands more. However, with new advancements in technology and the abundance of smartphones, digital solutions have presented new opportunities to contribute to public health epidemic response.

Digital contact tracing first emerged as a way to speed up the traditional contact tracing process and supplement public health's efforts by using smartphone technology for tasks like case management, symptom monitoring, and proximity tracking [6]. Exposure notification (EN) is a subset of digital contact tracing and is not meant to replace the traditional contact tracing infrastructure and process. EN refers to part of the contact tracing process in which close contacts of a confirmed case are informed that they have been exposed to a case. A variety of technologies and approaches have been used in different countries and states, each with different approaches to privacy protection and system capabilities. EN uses the Bluetooth Low Energy (BLE) technology built into smartphones to detect mutual proximity, without recording location information [7]. This method assumes proximity, measured by signal strength, as a proxy for distance (reflecting the CDC's distance per time quantification of meaningful exposure to define a close contact) while still preserving user privacy.

On April 7, 2020, University of Washington (UW) researchers and other collaborators released Privacy Sensitive Protocols and Mechanisms for Mobile Contact Tracing (PACT), proposed protocols for how to best utilize mobile phones to supplement contact tracing while preserving privacy and minimizing risks [8]. This guidance had some influence on Google and Apple's exposure notification (GAEN) protocol, released May 20, 2020 [9]. GAEN generates random identifiers every 10-20 minutes (Figure 1). Strength of the BLE attenuation is used to approximate a 6-foot distance for 15 minutes. This allows a device to determine whether another it has been "exposed" to another device and exchange identifiers with another phone. Understanding of appropriate configuration settings is still evolving. The recorded identifiers do not contain any information about the user or the location and are stored on the device for 14 days. Both the time and distance for determining an exposure can be changed. When a user tests positive for COVID19 , local public health authorities share a verification code that, when voluntarily entered into that user's smartphone, causes the device to upload to a central server the coded identifiers it has broadcast over the past 14 days. These anonymized identifiers are then downloaded periodically 
by other devices, who compare them to their own record of which device codes they have been "exposed to," based on a combination of signal strength and time. A match generates a notification of possible exposure on the receiving device. This leads to rapid contact notification, notification of contacts who could be unknown to the case and would not be reported during a case interview and helps combat recall bias. Early modeling suggested that with only a $15 \%$ adoption rate, infections could be reduced by $8 \%$ and deaths by $15 \%$ [10].

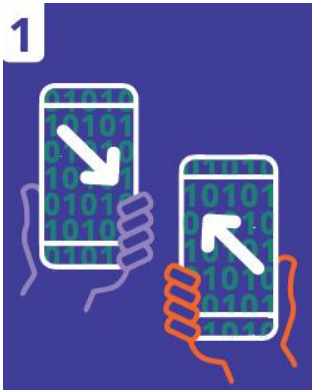

When two people

using WA Notify on

their smartphones

are near each other,

their phones exchange

random codes using

Bluetooth. The code

is completely

anonymous, with

no location tracking

or exchange of

personal information.
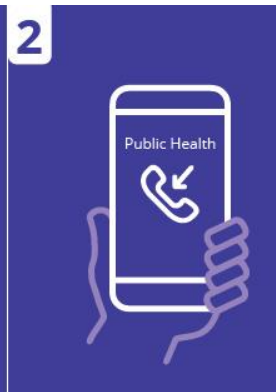

When someone in Washington tests positive and public health reaches out to them, they will be asked if they have WA Notify and given a verification code.
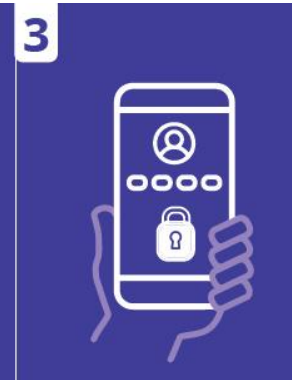

The person who tests positive then enters the verification code into WA Notify.

This is voluntary.
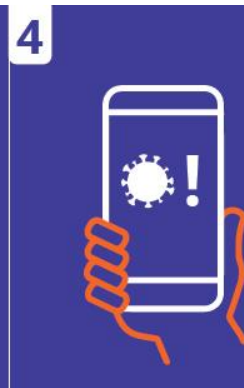

Anyone who also has WA Notify and has been near the user who tested positive for a significant length of time in the last 14 days will receive an anonymous alert that they may have been exposed to COVID-19.
5

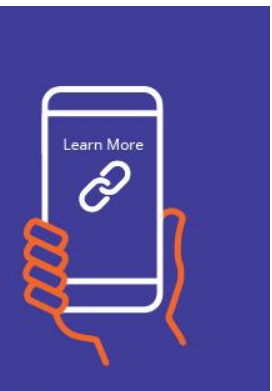

Notifications have a link to information about what to do next to protect yourself and others. They do not contain any information about who tested positive or where the exposure may have happened.

Figure 1. WA Notify uses Google Apple Exposure Notification technology to inform users of potential exposures [23].

Currently, over 20 states and U.S. territories have adopted GAEN technology to help slow the spread of COVID-19 [11]. However, Washington State (WA State) is an important case study for several reasons. WA State was the first U.S. state to report a COVID-19 infection on January 20, 2020 and one of the earliest states to be hit hard by the growing epidemic [12]. WA State was one of the earliest states to consider adopting the smartphone-based EN technology to support COVID19 responses, and sequentially developed and evaluated a locally developed app based on the PACT protocol, the use of an open-source GAEN app, and the implementation of WA Notify as a configuration of the Google/Apple Exposure Notification Express (ENX) embedded capabilities in iOS and Android phones [13]. Considerations included strong, thoughtful collaboration and planning between the WA State DOH, the UW and Microsoft Research, robust data collection, and pilot testing. These aspects of planning and rollout may have contributed to the success and rapid adoption observed in WA State compared with other states. This report will describe the implementation of Bluetooth ENX technology in WA State, from the planning and decisionmaking to the pilot testing, adoption, and early adjustments made (Figure 2). These lessons may be useful in evaluating the success and value of this technology, for other states still considering adoption of EN technology and in considering how to best implement similar solutions in the future. 


\section{WA NOTIFY IMPLEMENTATION TIMELINE}

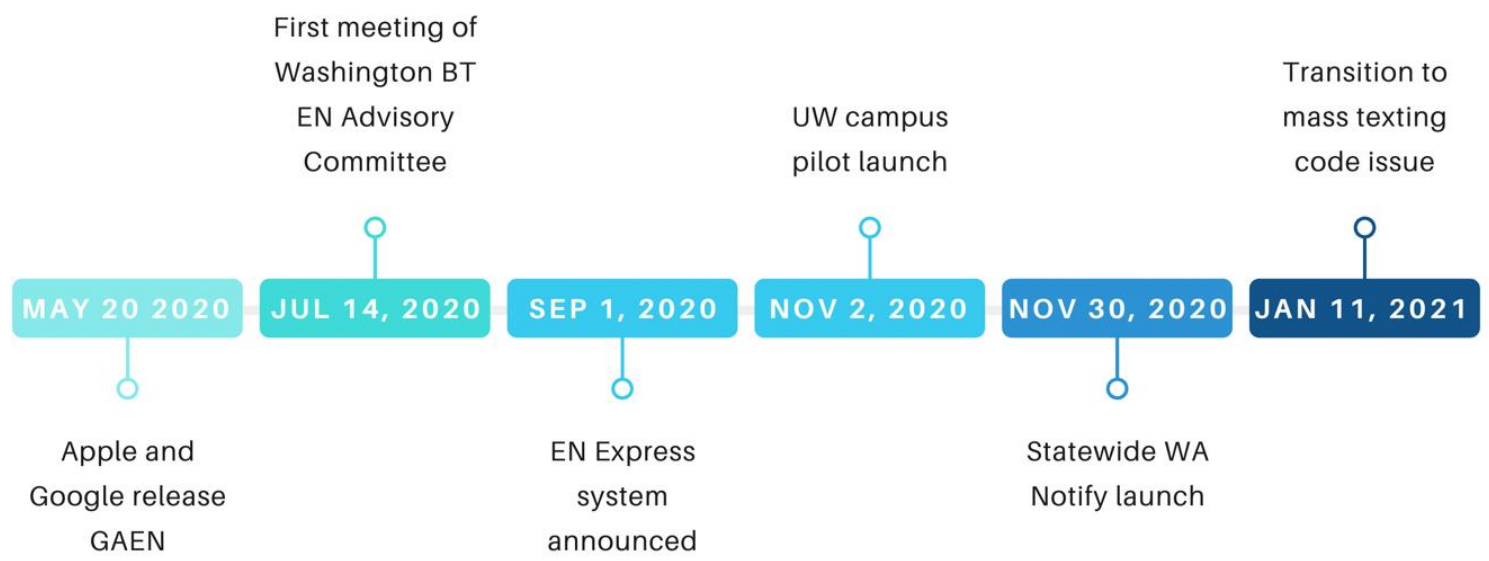

Figure 2. WA Notify planning, piloting, and implementation timeline and major milestones.

\section{Pre-implementation planning}

\section{Early design and decision making}

After the release of GAEN at the end of May 2020, and the anticipation of the second wave of COVID-19 cases after the initial spring peak, Governor Jay Inslee and WA State DOH leadership convened the COVID-19 Exposure Notification Advisory Committee to provide guidance, oversight, and recommendations regarding the implementation of this technology. At the time, no other state had formed a committee to oversee their BT EN work and make recommendations.

The committee, formed in June 2020, consisted of experts in key areas including mobile technology, public health, outbreak investigation, ethics, equity, and security. The committee also included representatives from community stakeholders. The committee met biweekly until midOctober and discussed various concerns and considerations about the use of this technology.

Recommendations from the committee included making accessibility and equity a priority, with many concerned that technology-based solutions may exacerbate existing disparities. These recommendations played a large role in informing ongoing evaluation work. The committee was also a space for representatives from important communities in the state, such as farm workers, people with disabilities, and tribes, to contribute their voices and ask questions. Ultimately, Governor Inslee and WA State decided to move forward with the use of BT technology for a statewide EN implementation for a smartphone.

\section{WA Notify pilot testing}

Near the end of summer 2020, the UW decided to move many classes for the Autumn quarter online, but still allow for limited in-person classes. Several thousand students returned to campus to live in residence halls or Greek residences. The start of the Autumn quarter created a perfect opportunity to pilot test the existing BT implementation among students, staff, and faculty. The 
WA Notify team saw this as an opportunity to collect information about potential technical issues, concerns and questions users would have, and to help inform integration of the tool into public health workflow.

In early September 2020, Apple and Google announced EN Express (ENX), an option that allowed state public health authorities to use GAEN technology without having to dedicate time and resources to building out their own tools. Each state can tune the parameters of the tool to meet statewide needs. WA State chose to adopt this method and was the second state to launch an ENX tool, shortly after Colorado. The "days since exposure threshold" was set at 14 days. Parameters were also set to approximate 6-feet proximity based on Bluetooth attenuation strength for 15 minutes, consistent with DOH's definition of a close contact as per CDC protocols.

Since the start of the pandemic, the UW Environmental Health and Safety (EH\&S) department has acted as the public health agency for the university population in collaboration with the state and local public health authorities. For COVID-19 cases among students or staff on campus, EH\&S would conduct surveillance, case investigation, contact tracing, and outbreak investigations. The EH\&S contact tracing team was trained on how to register with the Association of Public Health Laboratories (APHL), generate verification codes, and provide the codes to pilot participants who tested positive during the case investigation interview. The verification code, once entered by the user into the tool loaded on a smartphone, would alert other pilot users who had been in close contact with the confirmed case within the past 14 days.

The pilot version of the EN implementation was launched for the UW student population on November 2, 2020, and for employees on November 19, 2020. The UW Marketing and Communications department sent out invitation emails to eligible students and employees with information about the pilot and instructions on how to participate. Both Apple and Android users had a more complex installation process for the pilot than latter users had in the statewide deployment. Apple users were required to download a profile before downloading the tool, and Android users were given a link to the hidden tool on the Google Play Store. Supporting documentation including background information about the tool and an FAQ page were made available.

During the initial recruitment period, 23,324 students were invited to participate via email. This invitation was limited to students living in campus housing, Greek housing, or attending classes in person. Of those invited, 4,140 (18\%) clicked the link and were directed to the pilot website, and $2,238(54 \%)$ of those started to download the tool. The majority of download attempts used the Apple profile (1,771, 79\%) compared to the Google Play Store (467, 21\%). Among students who started a download, only 1,490 (65\%) completed the installation of the tool, resulting in an overall campus adoption rate of $6 \%$.

Adoption patterns were similar in the employee group. On November 19th, 37,128 employees were invited to participate, with 3,522 (9\%) people visiting the website. Out of these, 2,087 (80\%) initiated the tool download and 1,480 (71\%) of them successfully downloaded the tool, resulting in an employee adoption rate of $4 \%$. The adoption patterns indicate that each additional step of download decreased the level of user engagement. Providing users with direct access to tool download would likely increase adoption. Engagement and activations were highest directly after 
the initial invitation on November $2^{\text {nd }}$, with another increase after a reminder email to students on the $10^{\text {th }}$ (Figure 3 ). Cumulative installations also increased directly after staff and faculty were invited to participate on the $17^{\text {th }}$.

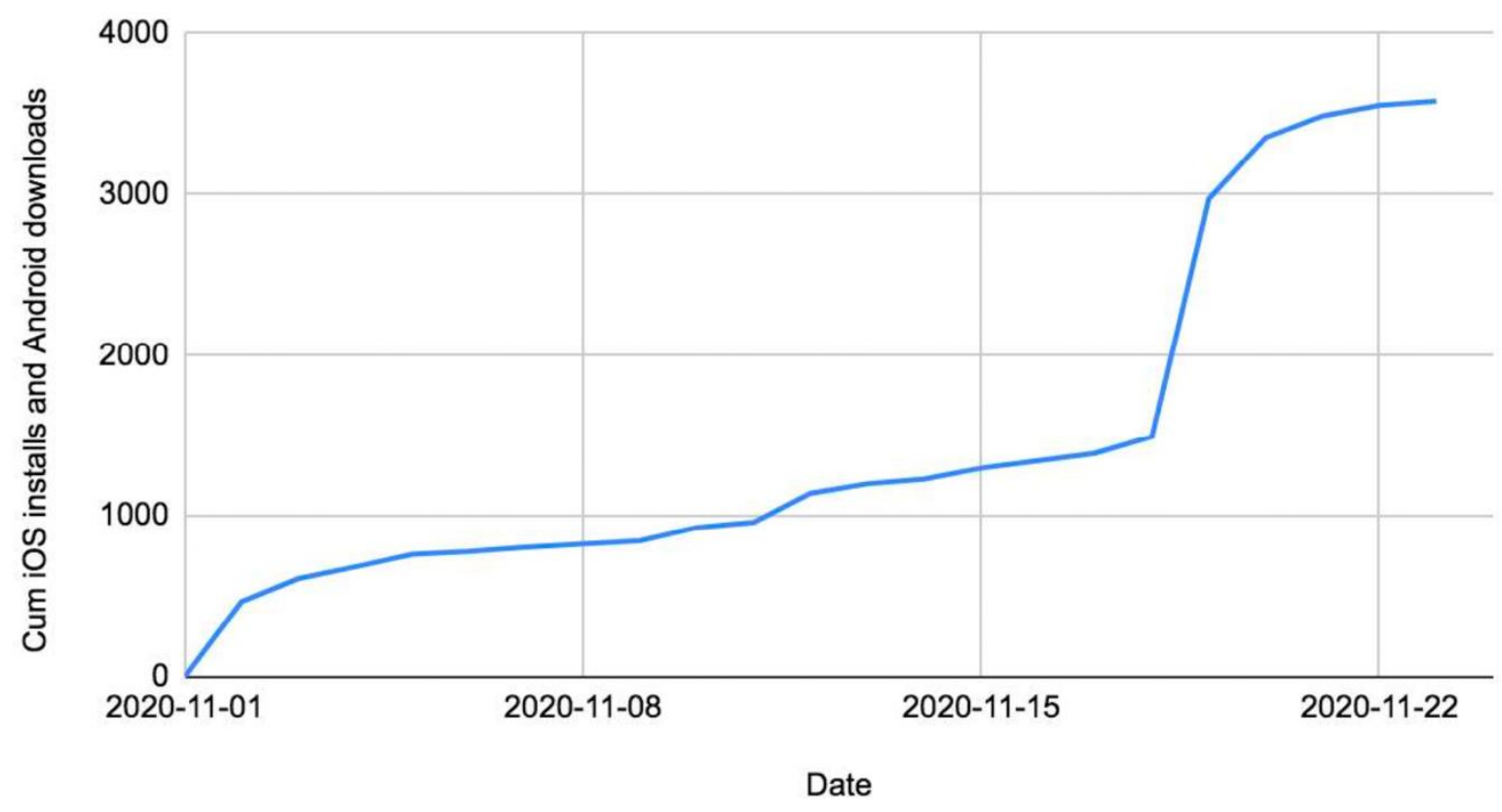

Figure 3. Cumulative WA Notify activations during University of Washington campus pilot over time, beginning November 2, 2020.

Feedback and user requests were tracked and analyzed by the UW pilot team. Questions and comments from pilot participants focused primarily on download and activation issues as well as questions about device and version compatibility. These comments and troubleshooting details helped shape the FAQs and other educational materials for the statewide launch.

\section{WA Notify integration with public health}

WA Notify relies partly on the existing public health and case investigation infrastructure to issue codes to positive cases. Thus, it was important to have buy-in and appropriate training and integration of this process before statewide implementation in a way that would not be overly burdensome.

In WA State, Local Health Jurisdictions (LHJs) manage contact tracing and case investigation for residents of their jurisdictions, with support from DOH provided as needed. Some state LHJs use the state's Case Risk Exposure and Risk Surveillance Tool (CREST), a tool developed in the summer of 2020 to facilitate case interviews and data management, and other LHJs use a platform of their choosing. LHJs were provided with training and scripts on how to ask if a case was a WA Notify user. Interviewers generated the 8-digit verification code and walked the user through the process of entering it into the tool. These codes expire after 15 minutes. Training materials differed between CREST and non-CREST LHJs, as code generation was integrated into the CREST 
platform. Case investigation interviews included additional questions regarding whether cases had an EN tool on their smartphone and if receipt of an EN was a reason for getting tested.

\section{Statewide implementation}

WA Notify became available across the state on November 30, 2020. The media and public relations plan for the launch of WA Notify consisted of four stages: initial launch, milestone announcements at certain adoption increments, New Year's reminders, and testimonials from users and endorsements from notable public personalities. The initial launch included a press conference by the governor, radio spots, social media posts, educational videos, and fact sheets. These media assets were made available in both English and Spanish.

The initial launch also included push notifications sent to iOS devices on November 30, alerting Apple users to the tool's availability. Several more notifications were pushed to iOS and Android devices throughout early December. To increase its reach, WA Notify was made available in 29 different languages. Initially, the tool was only available to those with iOS version 13.5 or later, and Android Version 6 or above. iOS compatibility was later expanded to version 12.5 in December to include older models of Apple devices.

Activations were high immediately following the launch, with over one million total installations of WA Notify within the first week (Figure 4). By the beginning of April, there were 1.9 million activations in the state, $86 \%$ of which came from Apple devices. The large majority of activations occurred shortly after the initial launch, with a much slower increase over time. Given a population size of 7.6 million people, this resulted in an overall adoption rate of $25 \%$ of the total population in WA State [14].

However, smartphone ownership by state is difficult to ascertain. Assuming smartphone ownership in the state is equal to the overall American average of $85 \%$, the approximate adoption rate in smartphone owners in WA State is 29\% [15]. The original adoption target was at least $15 \%$, based on the University of Oxford's modeling estimates of adoption effectiveness [10]. By the end of February, WA State successfully surpassed the original goal for adoption. 


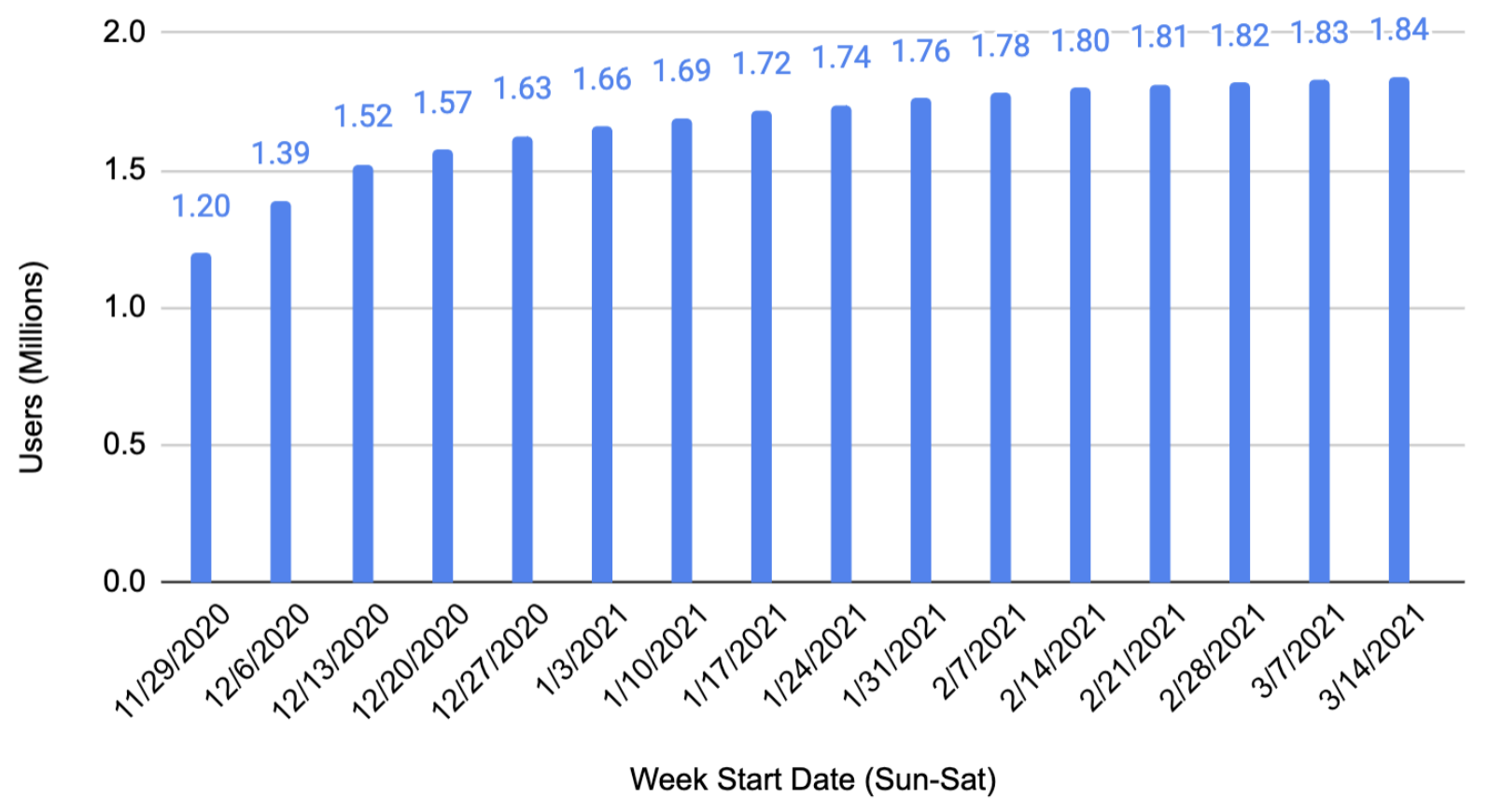

Figure 4. WA Notify total activations by week, starting the week of November 29, 2020, until the week of March 14, 2021.

Data on installations by iOS vs. Android, notifications, counts of verification codes, and other summary statistics were collected and tracked in an internal Google Sheets-based data repository and dashboard. This repository is updated daily with data from several sources. The UW WA Notify team continued to monitor and provide support to end users and answer their inquiries during the transition from the pilot to the statewide rollout, eventually transferred this responsibility to $\mathrm{DOH}$ at the end of December. User inquiries, requests and responses were documented and cataloged.

\section{Early adjustments: introduction of rapid automated texting}

After monitoring the initial adoption throughout the month of December, as well as COVID incidence overall, it became clear that high caseloads would put a strain on the contact tracing infrastructure and sheer numbers may cause delays in the process. After approval from leadership, planning began in December to develop a system for sending activation verification code messages to cases based on HL7 Electronic Lab Reportable (ELR) messages rather than relying solely on case investigators interviews. On January 11, 2021, WA Notify began using automated texting to issue verification codes (Figure 5). This method generates text messages in the APHL server that are sent to the phone number on file for all COVID-positive lab results each day. Each text includes an individualized code and information on what to do next. The codes expire after 24 hours, however, codes can still be issued during the case investigation if necessary. 


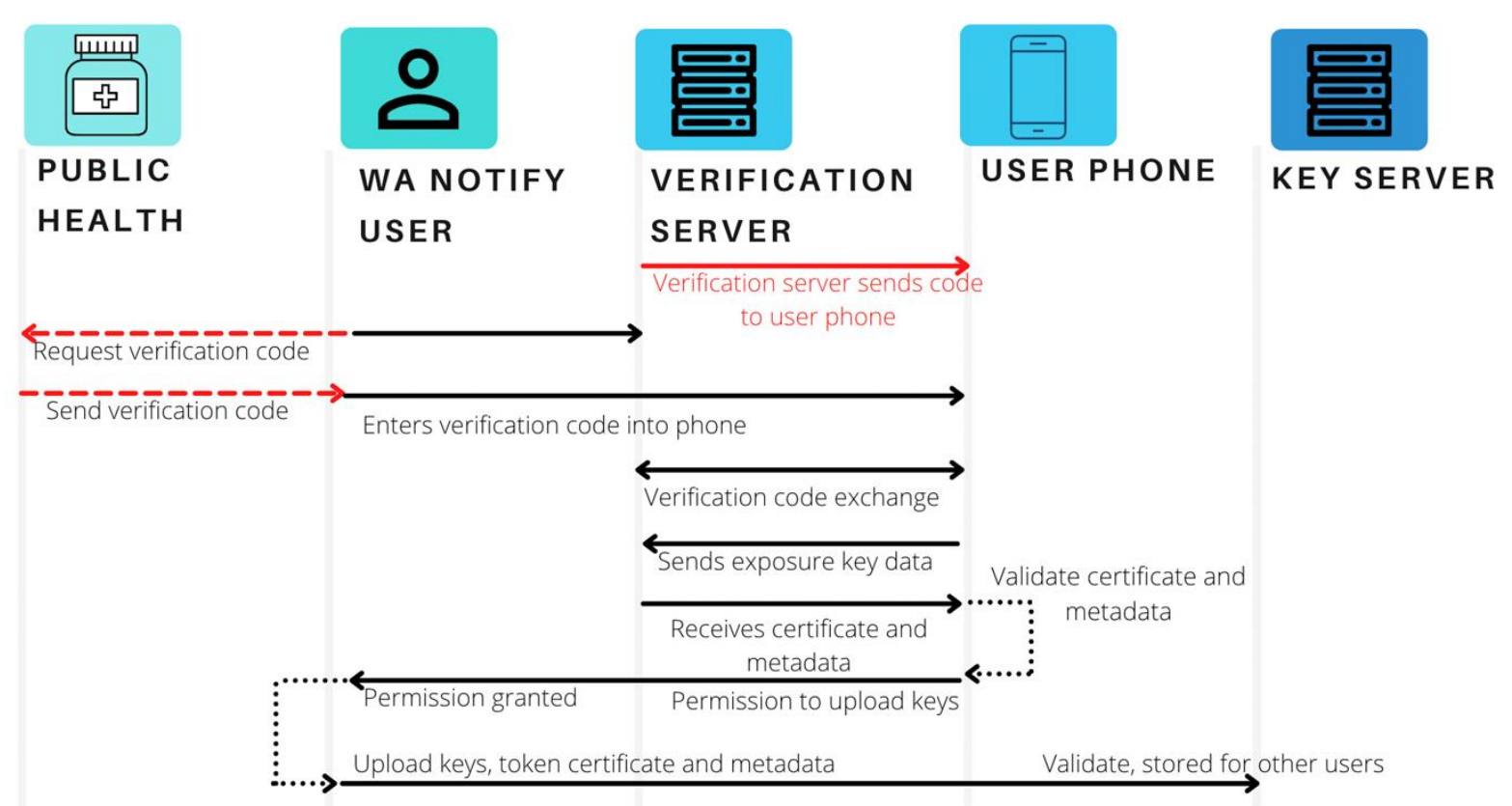

Figure 5. WA Notify verification code generation and notification process [24]. Red arrows indicate changes in the process when automated texting is used. Red dashed lines indicate that public health does not need to manually request and send the user the verification code. Instead, the server generates codes that are automatically sent to users' phones via text message.

Getting codes to users who test positive as quickly as possible ensures that possible close contacts receive a notification sooner and can take appropriate actions. Automated texting also minimizes the involvement of public health personnel, which is especially critical when resources are limited. It results in less need for codes to be issued person-to-person during a case interview, and fewer calls to public health to ask about codes, as seen in Colorado. This method still allows for codes to be issued in CREST if necessary.

Risks of the transition to automated texting were weighed heavily against its benefits. Positive cases may receive the text before even being notified of their test result. Receiving the text may lead to confusion, especially by those who are not WA Notify users. Additionally, it is not known for sure that phone numbers provided can receive texts or that it belongs to the person who tested positive. These risks were carefully weighed, and mitigation strategies were prepared.

The transition to automated texting resulted in a large spike in the number of codes being issued (Figure 6). Before the switch, less than 1,000 codes were being issued per week. In the initial week of automated texting, over 32,000 codes were issued and $6.1 \%$ of these codes were claimed. In the following weeks, the number of codes issued decreased slightly and eventually leveled off to around 4,000-5,000 codes per week. The percent of codes claimed over the following weeks ranged from $6-10 \%$. 


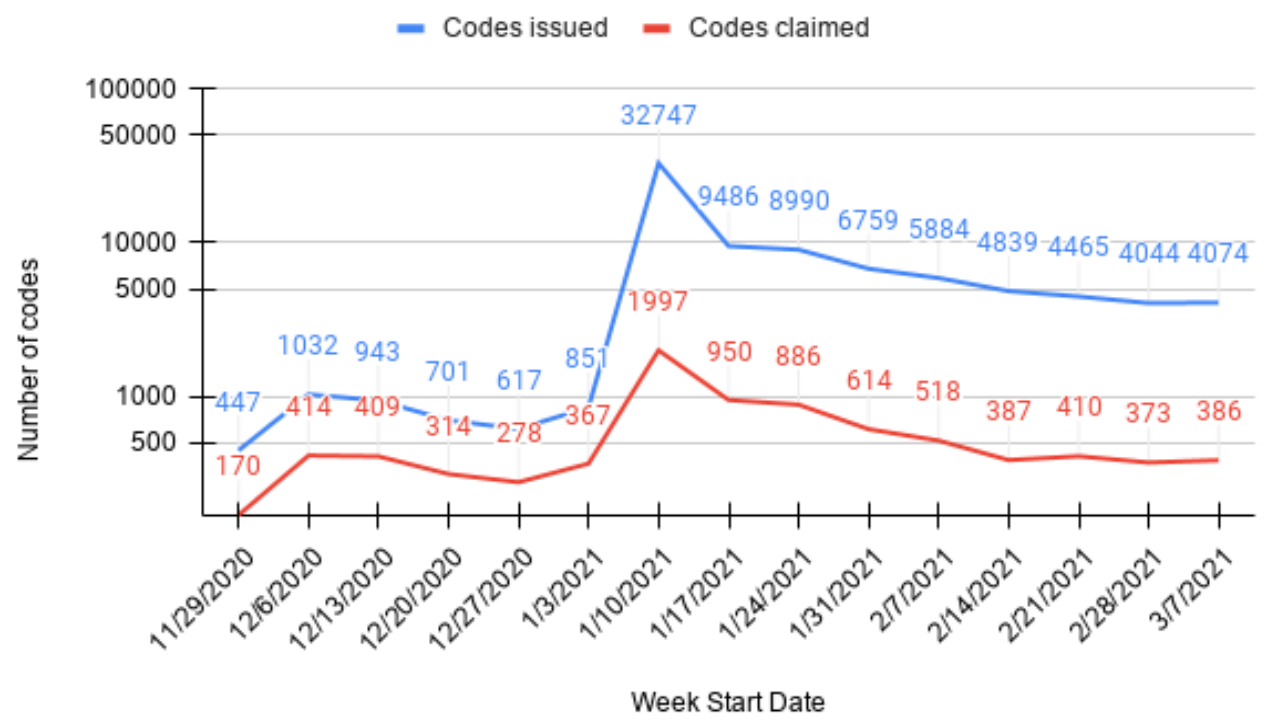

Figure 6. Number of WA Notify verification codes issued and claimed by users by week, from November 30, 2020, to the week of March 7, 2021. Automated texting of verification codes was enabled on January 11, 2021.

\section{Discussion}

After careful consideration and testing, WA State launched its Bluetooth ENX implementation, WA Notify, in November of 2020 to help mitigate the spread of COVID-19. As of April 2021, over 1.9 million WA State residents or $25 \%$ of the population and almost $30 \%$ of smartphones have activated the tool on their smartphones, making it one of the most wide-reaching EN tools in the country. Several reasons may underlie the success of its implementation: use of the ENX framework, the UW pilot study, and the formation of the Exposure Notification Advisory Committee.

Despite WA State being one of the earliest to consider the use of this technology, it was not one of the earliest states or countries to implement Bluetooth EN using the GAEN protocol. Taking the time to develop community input and advice took time but was important. The choice to use ENX, a free system embedded in the phone's operating system, provided substantial benefits, despite the delay in implementation. These included a considerable decrease in the need for upfront development of both app and infrastructure and decreased investment by public health. Also, the ENX system simplified both the user experience, once we were beyond the pilot phase, and allowed for easier push notifications to be sent directly to the device.

Several states launched their own EN tools prior to the availability of ENX and before WA State: six over the summer and four more by October. States who implemented early, like Alabama, Pennsylvania, and North Carolina, had adoption rates of less than $10 \%$ of the adult population in one month [16]. 
Other states using ENX also reported much higher adoption. Connecticut reported that their tool, COVID Alert CT, had been activated in about one-third of the state's population in January 2021 [17]. Washington D.C. reported that 53\% of residents had downloaded their DC CAN [18]. After adopting the ENX framework in February, Virginia doubled its number of activations in just a few weeks, bringing total adoption to $22 \%$ of the population [19]. However, it is difficult to compare adoption rates across different states due to different denominators (total population, adult population, smartphone owners) and lack of publicly available, up-to-date data.

Development of a BT ENX implementation for a smartphone such as WA Notify can be extremely expensive. UK NHS officials noted their tool costs total over $£ 35$ million (\$48M USD), and New York's cost around \$700,000 [20,21]. By using the free ENX platform, rather than investing in duplicative app development and customization, WA State was able to put $\$ 2$ million alone into a marketing and communications campaign [22]. Furthermore, the UW pilot enrolled almost 3,000 early adopters who formed an initial set of users when the system was expanded to statewide implementation. The pilot also allowed for user troubleshooting, allowed the team to understand what to expect from the launch, and increased public awareness of the tool.

The creation of the Exposure Notification Advisory Committee allowed for careful consideration of the risks and potential benefits of the use of the technology. While some states tried to launch their EN tools as quickly as possible without fully considering the implications of the decision, WA State's foresight paid off in the long run. Advisory Committee discussions, concerns, and recommendations helped form the foundation of the ongoing evaluation work.

\section{Limitations}

WA Notify and other GAEN-based solutions prioritize user privacy and do not collect information on location or identity. This may allow for higher acceptability and adoption, however, this also presents challenges. The impact of privacy on user adoption and the tradeoff ENX users prefer between privacy and functionality, has not been studied. Evaluation of WA Notify, its effectiveness, and user experiences are difficult because the identity of those receiving notifications is completely unknown. Since activating and using WA Notify is completely voluntary and anonymous, demographic information of the users is also not directly available. This makes it difficult to examine equity and adoption across groups, especially those disproportionately affected by COVID-19. Access to smartphone technology, as well as cell service, is unevenly distributed across the state, so WA Notify is not equally accessible to every single person in the state. Further work is needed to understand the impact of this inequity and how to mitigate it. Additionally, due to the novel nature of the technology, there is no previously existing framework on which to base the implementation and evaluation processes.

\section{Next steps}

WA Notify and similar tools promise to supplement the traditional contact tracing process and use existing technology to help mitigate the spread of COVID-19. However, whether Bluetooth exposure notification has been successful and delivered on these claims is still yet to be fully investigated. The privacy-preserving nature of the tool makes it even more difficult to answer this question. A research group at the UW has been planning and developing the evaluation of WA 
Notify since the summer of 2020. This robust evaluation addresses many of the limitations the tool brings by integrating a variety of data sources, including contact tracing data, population-based surveys, and interviews. The evaluation aims to assess WA Notify's public health value, unintended consequences, accessibility and equity, impact on contact tracing workflows, and more. The project will also investigate potential further uses of the technology. As we move forward, there is potential for public health agencies to adapt EN technologies to be used for diseases other than COVID-19.

\section{Conclusion}

The COVID-19 pandemic has posed unprecedented challenges to public health infrastructure and systems. Due to a lack of funding and resources, public health agencies are often slow to adapt technologically. This crisis, however, has led to public-private partnership and engagement that has never before occurred on such a large scale.

Bluetooth EN is a truly novel approach to controlling the age-old threat of pandemics. Partnership with multistate shared efforts and integrated ENX solution led to high adoption rates and cost savings that could be applied to outreach and evaluation. The lessons learned from the process of launching WA Notify and similar population-based interventions may be useful for future tools and technology. Digital tools and technology have the potential to play a further role in supporting public health, whether it comes to communication, immunization, or public outreach.

\section{Acknowledgments}

We would like to thank investigators from the University of Washington Paul G. Allen School of Computer Science and Engineering and Microsoft Research, whose work established the feasibility of BT EN and GAEN within WA State and enabled the subsequent state-wide adoption of WA Notify. We also thank University of Washington leadership for their support of the WA Notify pilot work on the university campus and the campus pilot technical team, notably Daniel Lorigan and Jenney Lee. We acknowledge the contributions of team members in scoping and providing feedback on this manuscript, notably Courtney Segal, Iris Jia and Tyler Bonnell. This project was supported through an inter-agency agreement between Washington Department of Health and the University of Washington.

\section{Financial Disclosure}

The authors do not have any financial disclosures to report.

\section{References}

1. Mooney G. 2020. "A Menace to the Public Health" — Contact Tracing and the Limits of Persuasion. N Engl J Med. 383, 1806-08. PubMed https://doi.org/10.1056/NEJMp2021887

2. CDC. Case Investigation and Contact Tracing : Part of a Multipronged Approach to Fight the COVID-19 Pandemic [Internet]. Centers for Disease Control and Prevention. 2020 [cited 
2021 Mar 29]. Available from: https://www.cdc.gov/coronavirus/2019-ncov/php/principlescontact-tracing.html.

3. Swanson KC, Altare C, Wesseh CS, et al. 2018. Contact tracing performance during the Ebola epidemic in Liberia, 2014-2015 [Internet]. PLoS Negl Trop Dis. 12 https://www.ncbi.nlm.nih.gov/pmc/articles/PMC6152989/. Accessed Mar 29, 2021. PubMed https://doi.org/10.1371/journal.pntd.0006762

4. Boylston A. 2014. John Haygarth's 18th-century 'rules of prevention' for eradicating smallpox. J R Soc Med. 107, 494-99. PubMed https://doi.org/10.1177/0141076814557198

5. Shankar AG, Janmohamed K, Olowokure B, et al. Contact Tracing for Influenza A(H1N1)pdm09 Virus-infected Passenger on International Flight - Volume 20, Number 1January 2014 - Emerging Infectious Diseases journal - CDC. [cited 2021 Mar 29]; Available from: https://wwwnc.cdc.gov/eid/article/20/1/12-0101_article.

6. CDC. Digital Contact Tracing Tools [Internet]. Centers for Disease Control and Prevention. 2020 [cited 2021 Apr 12]. Available from: https://www.cdc.gov/coronavirus/2019$\mathrm{ncov} / \mathrm{php} /$ contact-tracing/contact-tracing-plan/digital-contact-tracing-tools.html.

7. Sattler F, Ma J, Wagner P, et al. 2020. Risk estimation of SARS-CoV-2 transmission from bluetooth low energy measurements. npj. Digit Med. 3, 1-4. PubMed https://doi.org/10.1038/s41746-020-00340-0

8. Chan J, Cox L, Foster D, et al. 2020. PACT: Privacy-Sensitive Protocols And Mechanisms for Mobile Contact Tracing. Q Bull Comput Soc IEEE Tech Comm Data Eng. 43, 15-35.

9. Google. Exposure Notification API launches to support public health agencies [Internet]. Google. 2020 [cited 2021 Mar 29]. Available from: https://blog.google/insidegoogle/company-announcements/apple-google-exposure-notification-api-launches/.

10. Abueg M, Hinch R, Wu N, et al. 2021. Modeling the effect of exposure notification and non-pharmaceutical interventions on COVID-19 transmission in Washington state. npj. Digit Med. 4, 1-10. https://doi.org/10.1038/s41746-021-00422-7

11. O’Neill PH, Ryan-Mosley T, Johnson B. A flood of coronavirus apps are tracking us. Now it's time to keep track of them. | MIT Technology Review [Internet]. MIT Technology Review. 2020 [cited 2021 Mar 29]. Available from: https://www.technologyreview.com/2020/05/07/1000961/launching-mittr-covid-tracingtracker/.

12. Holshue ML, DeBolt C, Lindquist S, et al. 2020. First Case of 2019 Novel Coronavirus in the United States. N Engl J Med. 382, 929-36. PubMed https://doi.org/10.1056/NEJMoa2001191

13. COVID Shield [Internet]. GitHub. [cited 2021 Apr 9]. Available from: https://github.com/CovidShield. 
14. US Census Bureau. U.S. Census Bureau QuickFacts: Washington [Internet]. QuickFacts. 2019 [cited 2021 Mar 29]. Available from: https://www.census.gov/quickfacts/WA.

15. Pew Research Center. Demographics of Mobile Device Ownership and Adoption in the United States [Internet]. Pew Research Center: Internet, Science \& Tech. 2021 [cited 2021 Apr 12]. Available from: https://www.pewresearch.org/internet/fact-sheet/mobile/.

16. De La Garza A. People Are Finally Downloading COVID-19 Exposure Notification Apps. Will They Make a Difference? [Internet]. Time. 2020 [cited 2021 Mar 29]. Available from: https://time.com/5921518/covid-exposure-notification-apps/.

17. Office of Governor Ned Lamont. Governor Lamont Announces Connecticut's COVID-19 Contact Tracing App Has Been Activated on More Than One Million Mobile Devices [Internet]. CT.gov - Connecticut's Official State Website. 2021 [cited 2021 Mar 29]. Available from: https://portal.ct.gov/Office-of-the-Governor/News/Press-Releases/2021/012021/Governor-Lamont-Announces-Connecticuts-COVID-19-Contact-Tracing-App-HasBeen-Activated.

18. Hannon S. Exposure Notifications: end of year update [Internet]. Google. 2020 [cited 2021 Mar 29]. Available from: https://blog.google/inside-google/covid-19/exposure-notificationsend-year-update/.

19. Pegoraro R. COVID-19 exposure warnings for iPhone, Android phones: Apps still await widespread adoption [Internet]. USA TODAY. 2021 [cited 2021 Mar 29]. Available from: https://www.usatoday.com/story/tech/columnist/2021/02/24/covid-exposure-notificationapps-iphone-android-contact-tracing/4569421001/.

20. Downey A. Total cost of NHS contact-tracing app set to top $£ 35$ million [Internet]. Digital Health. 2020 [cited 2021 Mar 29]. Available from:

https://www.digitalhealth.net/2020/09/total-cost-of-nhs-contact-tracing-app-set-to-top-35million/.

21. Leswing K. States are finally starting to use the Covid-tracking tech Apple and Google built — here's why [Internet]. CNBC. 2020 [cited 2021 Mar 29]. Available from: https://www.cnbc.com/2020/10/03/covid-app-exposure-notification-apple-google.html.

22. Stiffler L. Washington state launches COVID-19 exposure notification app using Google and Apple technology [Internet]. GeekWire. 2020 [cited 2021 Mar 29]. Available from: https://www.geekwire.com/2020/wa-notify-covid-exposure-app/.

23. Notify WA. Washington State Department of Health [Internet]. [cited 2021 Mar 31]. Available from: https://www.doh.wa.gov/Emergencies/COVID19/WANotify.

24. Apple. Supporting Exposure Notifications Express |Apple Developer Documentation [Internet]. [cited 2021 Mar 30]. Available from:

https://developer.apple.com/documentation/exposurenotification/supporting_exposure_notifi cations_express. 